\title{
Shape and volume optimization of industrial parts
}

\author{
Juraj Beniak*, Miloš Matúš, Lubomír Šooš and Peter Križan \\ Slovak University of Technology in Bratislava, Faculty of Mechanical Engineering, Nam. Slobody 17, 81231 Bratislava, \\ Slovak Republic.
}

Global Journal of Engineering and Technology Advances, 2022, 10(01), 058-064

Publication history: Received on 13 November 2021; revised on 20 December 2021; accepted on 22 December 2021

Article DOI: https://doi.org/10.30574/gjeta.2022.10.1.0165

\begin{abstract}
In the present time, there are many challenges in the production of industrial parts. Due to the constantly rising prices of materials and energy, it is necessary to constantly look for ways to optimize production costs and optimize material consumption. There is great pressure on economical production, i. to produce products with the lowest costs given the expected and necessary properties. With the introduction of additive manufacturing technologies into practice and the production of parts for end use comes the introduction of methods for optimizing the shape of the part and the required amount of material for its production. We call this method Topological Optimization. The presented article describes the preparation of topologically optimized parts and a comparison of their strength properties with respect to the original and the original part.
\end{abstract}

Keywords: Topology optimization; Computer simulation; Component Strength; Shape optimization; Volume optimization

\section{Introduction}

Industries mainly focus on the manufacturing material quality and the final design outcome to remain competitive in this globalizing world. Topology optimization method is well suited tool for the prediction of improvised part designs in design process. An entire design of a required part can be done by using topology optimization throughout the process.

The addition of topology optimization with new CAD methods has been giving good results in achieving optimum structures automatically. Topology optimization stands first, usually to control the crude initial optimum layout, then it's followed by shape optimization to obtain the finely tuned layout. The interaction between these two optimization stages takes place by image processing tool which produce final topology and then sends the structure to shape optimizer. The evolution of same design model in all optimization steps plays a crucial role. The manufactures take the initial layout of the model and remove the small area where the stress is minimum which is ineffective to the model. This method is nothing but the structural reanalysis, which gives the stiffness to mass ratio [1-2].

The method topology optimization is a mathematical method, occurs just 20years before in 1988 for optimizing parts. It takes the part of design space which is called finite element mesh and increase the strength by minimizing the material. It is discovered by Bends and kikuchi in 1988 through homogenization to achieve topology optimization as the time passed; it also focused on density of material, in this paper it is mainly focused on density-based topology optimization. Sigmund and maute paper focus on density of material which is taken for reference. The main focus of

\footnotetext{
${ }^{*}$ Corresponding author: Juraj Beniak

Slovak University of Technology in Bratislava, Faculty of Mechanical Engineering, Nam. Slobody 17, 81231 Bratislava, Slovak Republic.
}

Copyright (C) 2022 Author(s) retain the copyright of this article. This article is published under the terms of the Creative Commons Attribution Liscense 4.0. 
topology optimization is material distribution within the preferred region. Topology optimization density-based problems are shown as giving the value of 0 for void and 1 for solid portion [3-4].

It represents as a solver for complex parts, but downgraded in high end analysis since it demands a predefined meshing. The stiffness of the model is dependent on the material balance in topology optimization. This method gained maximum popularity from past 2 years since it's able to get topology optimization in CAD software's and it is user friendly and affordable; also, to create strong part with less material topology optimization is suitable technology [5-6].

There is few possibilities how to make the optimization or what is the focus within the optimization process:

- $\quad$ Size optimization

- $\quad$ Shape optimization

- Topology optimization

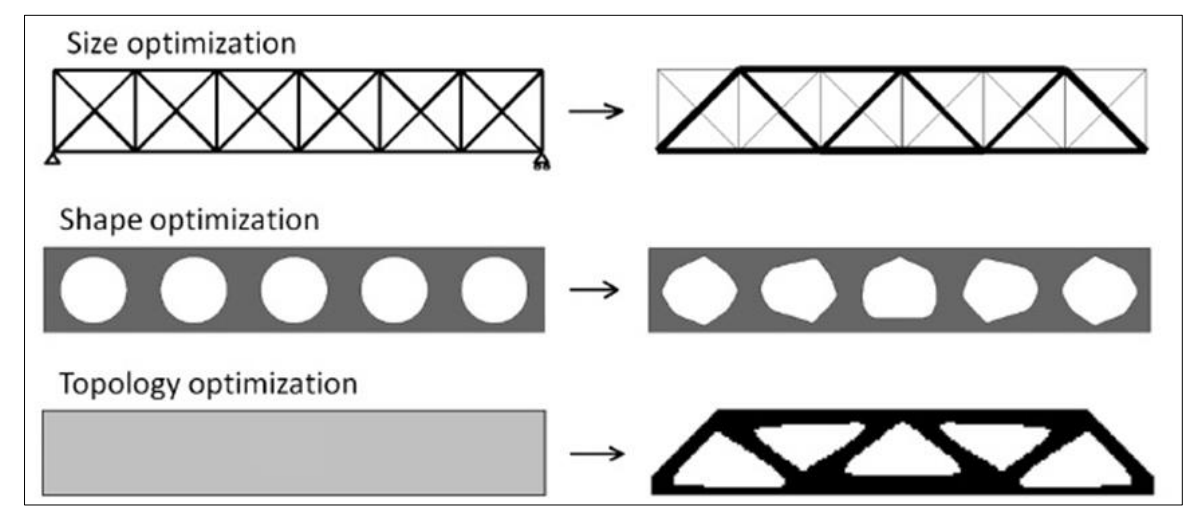

Figure 1 Types of part optimizations

Size optimization: - the main goal is to achieve good bar sections, it finds the best nodal positions of predefined nodes of the structures.

Shape optimization: - It gives the systematic frame work for optimizing structure by different physical and mechanical models. [7].

Topology optimization - reduces the size of material by taking the 3D design to achieve the possible effective design. This process doesn't give much importance to aesthetics, traditional ways or some other design process constraints which we use normally use in general designing. By defining the loading \& constraints automatically it will find out the required material to develop the load path.

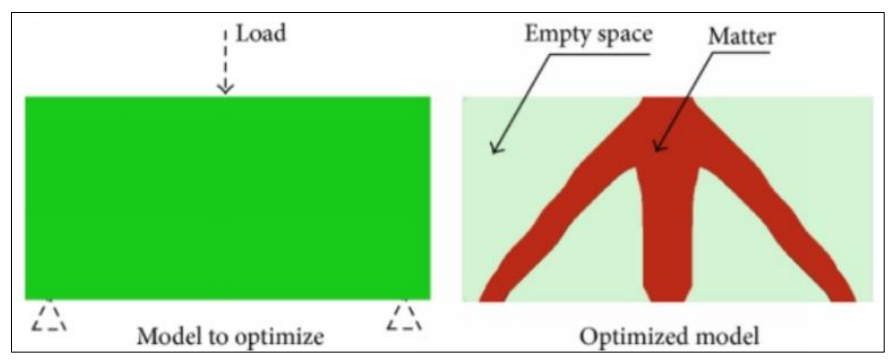

Figure 2 Illustration of optimization process [8]

The optimization is started by giving an initial input or basic idea. This is very important because usually faces nonconvex optimization problems which results in high local optimal topologies. Finet element analysis (FEM) will be performed based on initial design. Ad joint method is used to determine the sensitive analysis. After collecting the required information, the optimization process will be conducted on design to be updated. The design variable consists of a greater number of topology optimization problems that is usually non-linear optimization. The structural optimization consists of particular application called The Method of Moving Asymptotes (MMA), due to their flexibility and application in large scale problems these are well suited for density-based topology optimization. The optimization 
is checked for convergence after the design is updated to required criteria. This complete cycle keeps on repeating if the convergence criteria don't meet the required point. [7].

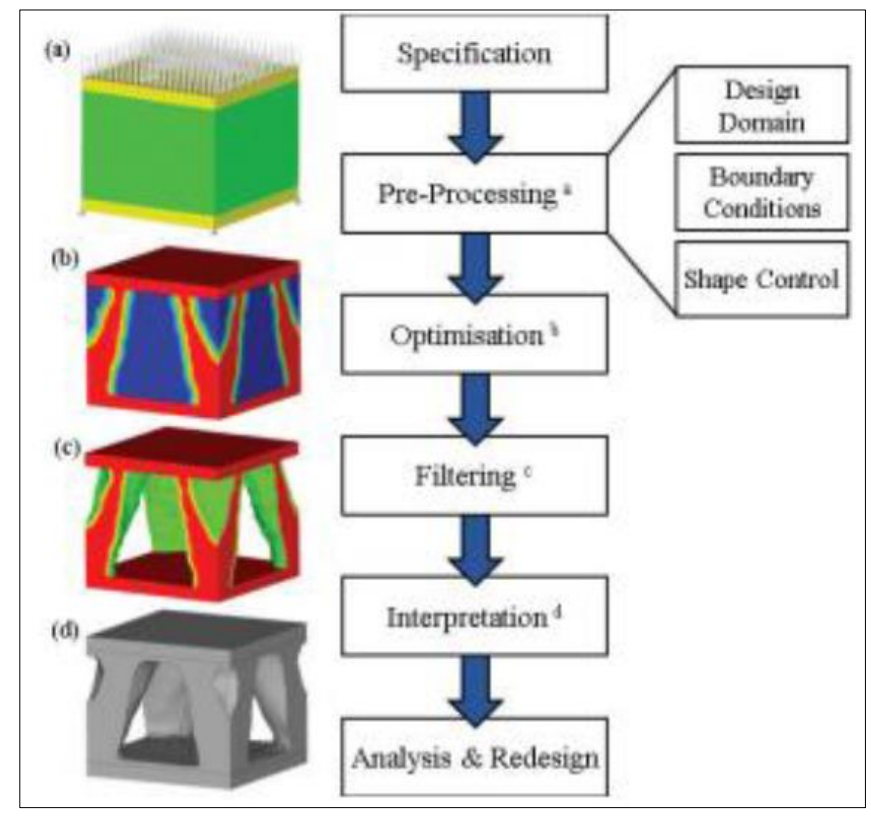

Figure 3 Work flow of topology optimization [9]

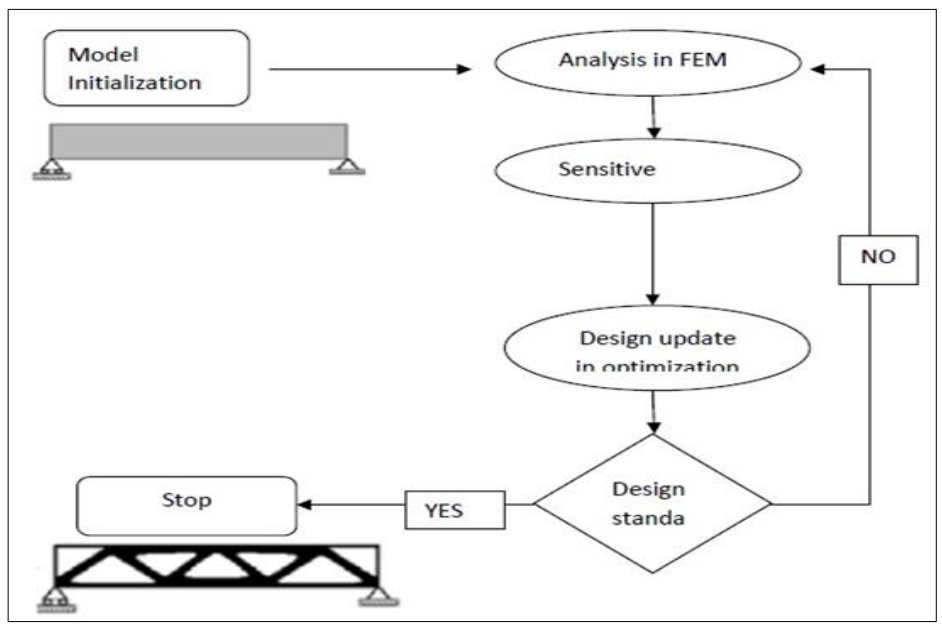

Figure 4 Flowchart of topology optimization process within software

Topology optimization is a gift to designers. It pushes to finish the maximum thinking of designers. It can be both additive and subtractive algorithm, but in this paper, we mainly focus on subtractive algorithm method. The software starts its work by changing a design space into mesh and then pushes to further simulation by taking the user defined loads and boundary condition. The software considers manufacturing constraints and stiffness of every cut-out element, this process continues until it meets the constraints of parts.

Then the software compares the initial shape and the shape undergone deformation with given load, to takes values of deviation. The point is defined for every element and can be alter at any required time. The designers can also change the geometry of the part at certain limits of manufacturing constraints, parameters like thickness, symmetry, direction etc. This shows that the optimized part can be manufacture except its unaesthetic shape.

The main constraints of topology optimization are;

- Maximum deflection

- Amount of mass needed to remove from the part 
- Manufacturing process

- Number of mechanical copies

The main goals of topology optimization are;

- Maximum displacement

- $\quad$ Stiffness to weight ratio

- Minimum mass of the part

\section{Material and methods}

On the Figure 5 is one of the general examples of a normal CAD part which is designed in Solidworks. The material used for this part is ABS. The part weighed $0.04256 \mathrm{Kg}$ under the load $4800 \mathrm{~N}$; further the stress analysis is completed using simulation that we can witness further in this paper.

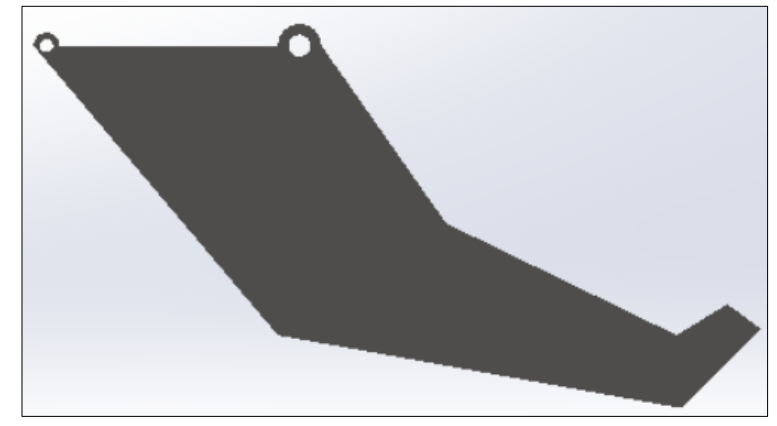

Figure 5 Industrial part - Original before optimization

Figure 6 represents the optimized part using topology optimization in solidworks, by using loads and boundary condition. In this part we can observe the reduction of material after optimization when compared to normal CAD part. The material is reduced up to $40 \%$ of the original mass and we used the same ABS material under the load $4800 \mathrm{~N}$, now the part weight reduced to $0.02763 \mathrm{Kg}$. This shows that the reduction of mass of the part can be achieved in topology optimization with feasible method. The stress analysis for the same part after the optimization has shown further in this paper to check the strength of the optimized part by comparing to normal CAD part.

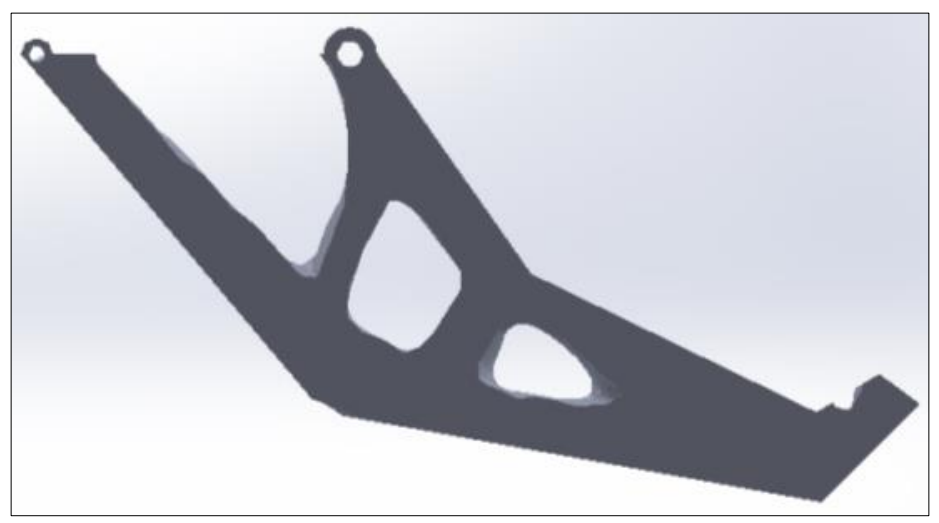

Figure 6 Industrial part - after optimization

In stress analysis we can conduct several tests using simulation. In this paper these tests are conducted on some of the simple CAD models before topology optimization and after topology optimization for the same CAD models and comparing both the results to identify the stiffness to mass ratio.

In simulation we can find out the static analysis to find out equivalent stress, strain and displacement of the designed part where stress in $\mathrm{N} / \mathrm{m}^{\wedge} 2$ (Von misses) and displacement in $\mathrm{mm}$. A sample part has been tested static analysis in 
Solidworks 2018 and further the same analysis is tested for optimized part to find out the comparison between normal CAD part and topology optimized part. The above normal part shows the static analysis with an initial weight.

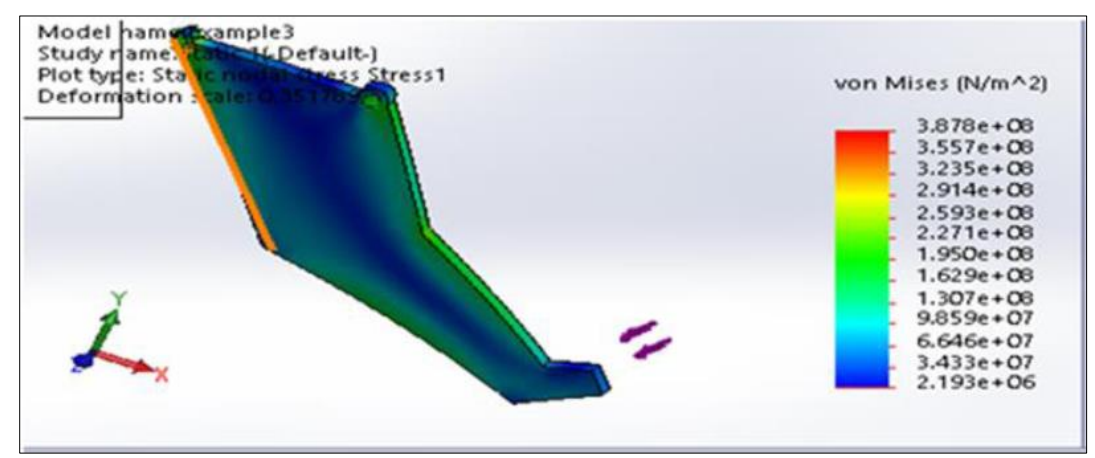

Figure 7 Stress analysis performed on normal CAD part

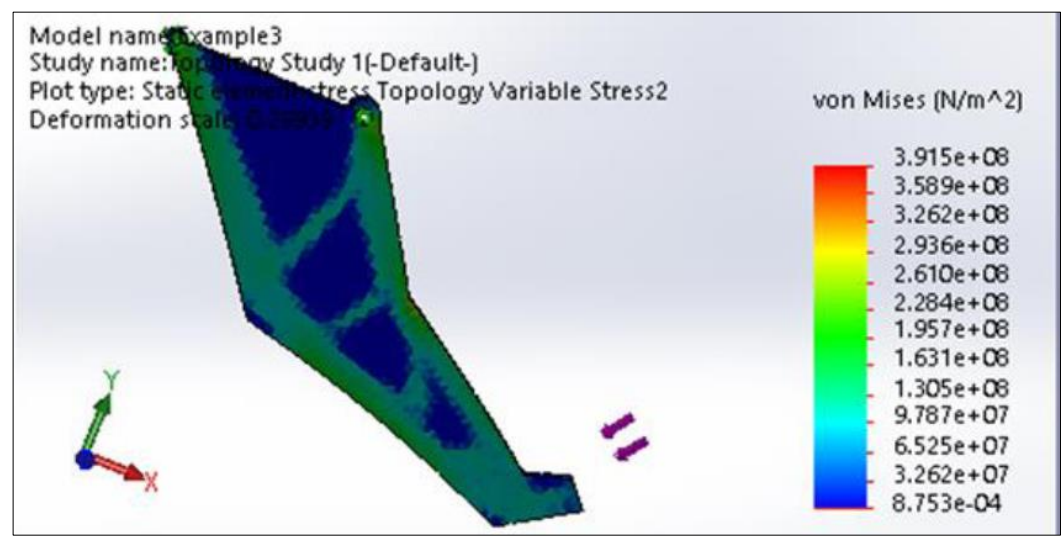

Figure 8 Stress analysis performed on optimized part

From the above figure we can compare the results of equivalent stress. The figure at the top shows the maximum and minimum stress of a normal CAD part and the below figure shows the maximum and minimum stress of an optimized part. The readings from the table says that the normal cad part holds bit more strength compared to optimized part, to overcome this we can increase the maximum stiffness to mass ratio by $10 \%$ so that it can withstand more strength than the normal part.

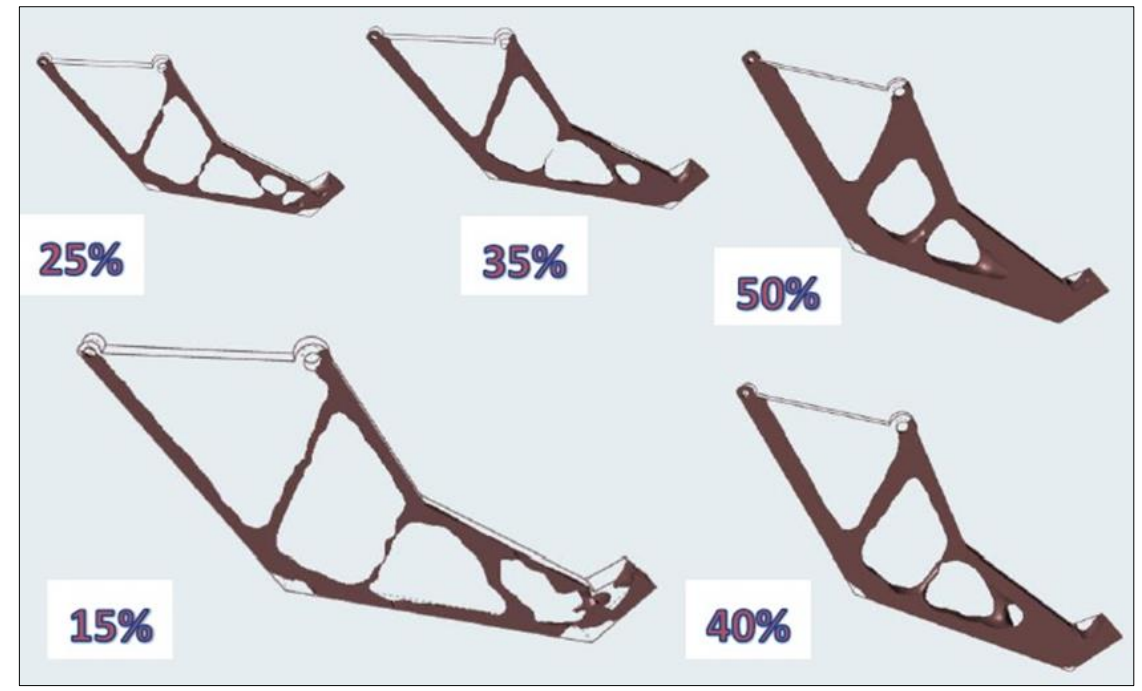

Figure 9 Illustration of shape of topology optimized parts with different rest of material 
The simulation of topology optimization needs before processing; that includes, applying boundary condition, creating some required design domains, controlling shape and performing accurate constraints for optimization. In this paper these conditions are undertaken through solid works software.

In the above figure we can observe the different percentage of mass reduction. We removed the material from $10 \%$ $50 \%$ to check the strength of the part. The main aim of this experiment is not just to save money by reducing its mass, but at the same time it should withstand the same strength or more than unoptimized part. In this percentage of mass reduction concept, the $10 \%$ mass reduction says that; it keeps just $10 \%$ of the overall mas and remove remaining $90 \%$ of its mass. We came across different mass reduction, as the reduction changes the values of the stress analysis also changes. In the above example that we took for different percentage of mass reduction, in this example we witnessed that as the mass reduced from $50 \%$ to $10 \%$ the strength of the part also decreased. The value of stress analysis on this part at $40 \%$ mass reduction is shown completely in next topic. The strength of the part before optimization increased, but if we remove more material regardless of the support and force automatically the strength of the part gradually decreases.

\section{Results and discussion}

The optimization takes place in a design space where we can remove the material that contain less stress area. In the above example as we keep on reducing the percentage of mass from 50, 40, 35, 25, 15, 10 the mass reduces from 0.04 , $0.024,0.021,0.014,0.0074,0.0041 \mathrm{Kg}$ respectively. The strength of the part increased more than the unoptimized part from $50 \%$ and up to $40 \%$ after $40 \%$ of mass reduction the part started to losing its strength than the normal part. This says that while performing topology we should give more attention towards the design space to remove material in less stress area. We can observe the reducing of material as the reduction of mass drops from $50 \%-10 \%$.

Table 1 Values of stress analysis with respect to Maximum stiffness to mass ratio

\begin{tabular}{|c|c|c|c|}
\hline Maximum stiffness to mass ratio (\%) & Mass (kg) & Displacement (mm) & Von mises stress (Mpa) \\
\hline 10 & 0.0041 & 3.195 & 31670 \\
\hline 15 & 0.0074 & 0.4506 & 4390 \\
\hline 25 & 0.014 & 0.2287 & 3131 \\
\hline 35 & 0.021 & 0.12 & 2699 \\
\hline 40 & 0.024 & 0.14 & 844.7 \\
\hline 50 & 0.030 & 0.077 & 956.5 \\
\hline 100 & 0.058 & 0.097 & 1024 \\
\hline
\end{tabular}

From the above table we can observe the stress analysis performed values on different maximum stiffness to mass ratio. This says that the unoptimized part have a von mises stress value of $1024 \mathrm{MPa}$ and a displacement of $0.097 \mathrm{~mm}$ with a mass of $0.058 \mathrm{Kg}$. As we can observe the other values which shows in table, we can select maximum stiffness to mass ratio of 50\%; since it contains more strength by seeing the stress analysis of $956.5 \mathrm{MPa}$ and a displacement of 0.077 with a mass of $0.030 \mathrm{Kg}$ for the same load and boundary condition. The max stiffness to mass ratio of $50 \%$ worth more than any other in this table, because of the reduced mass and less stress and displacement.

Table 2 Comparison of values for example 1 with and without topology optimization

\begin{tabular}{|l|c|c|c|}
\hline Properties & Original Part & Optimised (40\%) & Difference (\%) \\
\hline Von Mises Stress (Mpa) & 387,8 & 391,5 & 0,95 \\
\hline Displacement (mm) & 0,4838 & 0,5727 & 18,37 \\
\hline Mass (kg) & 0,04256 & 0,02553 & 40,01 \\
\hline Applied force (N) & 4800 & 4800 & \\
\hline
\end{tabular}




\section{Conclusion}

In this paper the main focus is on an innovative design tool topology optimization, by referring other published journal to gain knowledge on topology optimization and designing a simple CAD models as an example to perform stress analysis and topology optimization on those parts using software Solidworks.

The simple examples are designed in an CAD tool using Solidworks and then the part goes under stress analysis to check the strength of the part and the readings are noted. The material used for the examples is ABS. Topology optimization is performed on the same example and then again stress analysis readings are noted. From the values we gathered we can say that the examples that goes under optimization are quite stronger than the unoptimized parts, the maximum stiffness to mass ratio of optimized parts are $40 \%$ which is lesser in weight compared to unoptimized parts. Since the optimization took place only in design space, only the unwanted materials are removed where the stress area is less. The calculation part to remove material is automatically calculated by topology tool, it saves the most of the designer time.

To check the maximum stiffness to mass ratio, we have performed topology by several different percentage of 10,15 , 25, 35 and $50 \%$. From this test we can say that, while removing the material we should focus on the stress area. As we give less percentage it removes more area from the part that we can observe in the figure. If we neglect the manufacturing constraints the part will become weaker. Its better if we check for stress analysis of the part as we remove material to achieve less material and high strength.

\section{Compliance with ethical standards}

\section{Acknowledgments}

The paper is a part of the research done within the project APVV- 18-0527 "Development and optimization of additive manufacturing technology and design of device for production of components with optimized strength and production costs" funded by the Slovak Research and Development Agency.

\section{Disclosure of conflict of interest}

There are no conflict of interest.

\section{References}

[1] Yuksel, Osman. An overview on topology optimization methods employed in structural engineering. 2019; 5: 159-175.

[2] Ahmad A, Bici M, Campana F. Guidelines for Topology Optimization as Concept Design Tool and Their Application for the Mechanical Design of the Inner Frame to Support an Ancient Bronze Statue. Appl. Sci. 2021; $11: 7834$.

[3] Fiuk G, Mrzygłód M. Topology optimization of structures with stress and additive manufacturing constraints. Journal of Theoretical and Applied Mechanics. 2020; 58(2): 459-468.

[4] VB Hammer, N Olhoff. Topology optimization of continuum structures subjected to pressure loading, Struct Multidisc Optim. 2000; 19: 85-92.

[5] Jihong ZHU, Han ZHOU, Chuang WANG, Lu ZHOU, Shangqin YUAN, Weihong ZHANG. A review of topology optimization for additive manufacturing: Status and challenges, Chinese Journal of Aeronautics. 2021; 34(1): 91110 .

[6] Rozvany G. Aims, scope, methods, history and unified terminology of computer-aided topology optimization in structural mechanics. Struct Multidisc Optim. 2001; 21: 90-108.

[7] HAERTEL, JAN HENDRIK KLAAS. Design of Thermal Systems Using Topology Optimization. 4-5 and 24-25.

[8] AA Garcia-Granada, J Catafal-Pedragosa, HG Lemu. Topology optimization through stiffness/weight ratio analysis for a three-point bending test of additive manufactured parts.

[9] Dan Waltona, Hadi Moztarzadeh. Design and development of an additive manufactured component by topology optimization. 207. 